\title{
Thermal Cross-Talk in IC-Compatible Micromachined Infrared Thermopile Detector Arrays
}

Wu/Huaiwen

Delft University of Technology

Mekelweg 4, 2828 CD Delft, The Netherlands

\section{Introduction}

Arrays of thermal detectors are widely applied in optical instruments. IC technology enables the fabrication of large numbers of indentical thermal sensors. Thermal cross-talk between neighboring sensors is a common problem in these devices. This papaer discusses thermal cross-talk in ThermoElectric (TE) detector array on thin-film bridges for an infrared(IR) micro-spectrometer. In this system the disprsed IR spectrum from a different grating is projected onto a detector array[1]. The position of a spectral component within the IR spectrum is determined by the position of the element in the array, and the optical power within that part of the spectrum which is also the absorbed heat at that element. The temperature gradient at each element due to the absorbed IR radiation is detected using a thermopile between suspended absorber and the bulk silicon. Measuring temperature difference makes the integrated system insensitive to variations in chip temperature.

\section{Theory and model}

Conduction, convection and radiation are the three mechanisms of loss. The thermal transfer due to these three mechanisms acting on the TE element that is mechanically supported using a bridge structure is shown schematically in Fig 1. The dimension of bridge is $36 \mu \mathrm{m}$ in width and $650 \mu \mathrm{m}$ in length. The gap between elements is designed to have $10 \mu \mathrm{m}$. All three loss mechanisms should be considered when analysing the sensitivity of a single element. Due to the aspect ratio of an element (small sidewall area) only heat exchange between adjacent elements by conduction and convection is considered. Since the detector is packaged within the small encapsulated cavity, the behaviour of gas and temperature has a significant influence on the performance of detector. As is shown in this analysis, the gas conductivity determines the limit of what can actually be achieved by the design of the support structure and the structural separation of the elements.

Electrical analogies have been used for the simulation of the dynamic behaviour. The experimental results are presented to validate the simulations. Conclusions on the results and the effectiveness of the structural separation of TE detector elements on cross-talk, as compared to vacuum operation, are drawn in the last section.
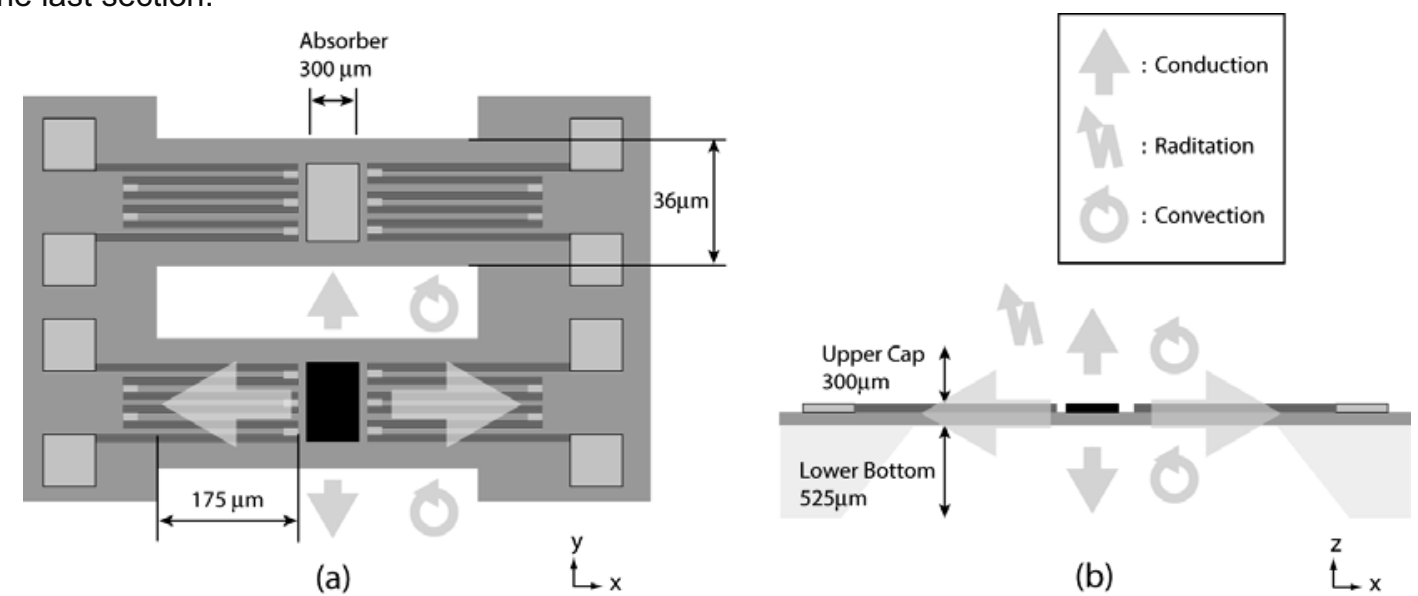

Figure 1 Heat transfer mechanisms on the thermopile bridge-shaped detector. (a) Two bridges are illustrated and the below bridge has the absorbed heat flux to transfer. (b) The cross-sectional view. 


\subsection{Thermal conductivity of air}

The relationship between thermal conductivity of air (gas) as the function of temperature and environment pressure is in Eqn. (1) [2]. The dimension of cavity in package is small enough in such a way the thermal conductivity of air as a function of pressure between two closed plates can be approximated into

$$
k_{\text {air }}=k_{0} \times \frac{1}{1+\frac{7.6 \times 10^{-5}}{p \times \frac{D}{T_{\text {avg }}}}}\left[\mathrm{Wm}^{-1} \mathrm{~K}^{-1}\right],
$$

where $k_{0}, p, D, T_{\text {avg }}$ denotes the thermal conductivity of air at room pressure and temperature, the pressure, the distance between the plates and the average temperature of the plates respectively.

Assuming the distance between the plates is $1 \mathrm{~mm}$, the average temperature is $300 \mathrm{~K}$ and the $k_{0}$ is $0.0284 \mathrm{~W} / \mathrm{mK}$

\subsection{Theoretical of the thermopile detector}

The infrared thermopile detector used is based on a Seebeck effect. Radiation incident on the detectors heats up the absorption region and results in temperature gradient, which is converted into a voltage. Analytical solutions for the heat conduction, which include convection and radiation losses, have been developed, by assuming the direction of heat transfer in only one direction [3-4]. There are three paths for the heat flow generated from radiant energy on middle of the bridge-shaped thermo-electric detector. The first path is the heat conduction through the solid materials that comprise the suspension. The equivalent thickness and equivalent thermal conductivity of multi-layers bridge can be expressed as:

$$
\begin{aligned}
& d_{e q}=\sum_{i=1}^{n} d_{i}[m], \\
& k_{e q}=\frac{1}{d_{e q}} \sum_{i=1}^{n} d_{i} k_{i}\left[W m^{-1} K^{-1}\right],
\end{aligned}
$$

where the $d_{i}$ and $k_{i}$ denotes the each thickness of thin film and each thermal conductivity of material respectively.

The second path is the convection loss. Since the space in the package contained the detector array is limited, the buoyancy forces of air cannot be larger than the viscous forces of air. The convection effect at which the air flow carries the heat from the detector surface to ambient can be neglected [5]. Except the loss due to air flow, the conduction loss of air from the surface of bridge to package enclosed plates should be taken into account. This loss is expressed as the convection coefficient in heat transfer equation. The last loss is due to the radiation effect and can be expressed as

$$
4 \sigma_{B} A\left(\varepsilon_{u}+\varepsilon_{l}\right) T_{a m b}^{3} \text {, }
$$

where $\sigma_{B}$ is Stefan-Boltzmann constant, $A$ is the emission area(equivalent to absorption area in element), $\varepsilon_{u}$ and $\varepsilon_{1}$ are the emissivities of the upper and lower layers of emission area respectively.

Based on the heat transfer theory, the Fourier equation can be expressed as follow:

$$
\rho C_{p} \frac{\partial T}{\partial t}+\nabla \cdot\left(-k_{e q} \nabla T\right)=Q-\rho C_{p} \nabla T,
$$

where $\rho$ is the density of material, $C_{p}$ is the specific heat capacity, $Q$ is the heat flux.

The dynamic response of thermal detector array is related to the thermal time constant of the detector. As a result, it is needed to calculate the equivalent heat capacitance of the bridge with materials on top and is expressed as:

$$
C_{e q}=\sum_{i=1}^{n} V_{i} \rho_{i} c_{i}\left[J K^{-1}\right],
$$

where $V_{i}, \rho_{i}, c_{i}$ denotes the volume of the material, density of the material and the specific heat capacity of the material.

The detector element has been expressed in terms of lumped elements and is shown in Fig 2. The structural parameters and dimensions of the TE element haven been taken as constant, while the air gap has been modeled as the variable component, due include the effect of environment pressure. Three TE elements are included in the figure. The heat conductivity of the main element along the bridge suspension is represented by the thermal resistance $R_{\text {main }}$, while the heat capacitance of the heated volume is represented by $C_{\text {main }}$. Thermal coupling via the air gap is represented by $R_{\text {gap } 1}$ and $C_{\text {gap } 1}$. 
Similarly, the coupling to the next element is represented by $R_{\text {gap2 }}$ and $C_{\text {gap2 }}$. Due to symmetry only the part left or right from the main element needs to be considered. The thermal parameters used for simulation is listed in the Table I.

TABLE I Thermal properties of material used for the simulation.

\begin{tabular}{c|c|c|c|c}
\hline & Thickness $[\mu \mathrm{m}]$ & Thermal conductivity $\left[\mathrm{Wm}^{-1} \mathrm{~K}^{-1}\right]$ & Specific heat $\left[\mathrm{Jkg}^{-1} \mathrm{~K}^{-1}\right]$ & Density $\left[\mathrm{kgm}^{-3}\right]$ \\
\hline Silicon ntride & 0.9 & 1.55 & 170 & 2440 \\
\hline Polysilicon & 0.3 & 25 & 700 & 2329 \\
\hline Silicon & 21 & 130 & 700 & 2329 \\
\hline
\end{tabular}

The modulus of the thermal response of the heated (main) pixel as a function of packaging pressure is shown in the Fig 3 and is normalized at the response for air pressure at $10^{-5}$ Bar. The simulation result shows the DC sensitivity is improved by decreasing the air pressure and is in agreement with literature. Moreover, all curves have the same cut-off frequency in the simulation. The modulus of the thermal cross-talk is expressed in terms of the temperature increase of the neighboring element relative to that of the heated element itself. The dynamic response of cross-talk to the side1 element (Figure 4 ) at $0.1 \mathrm{mBar}$ pressure is shown in the Fig 4.

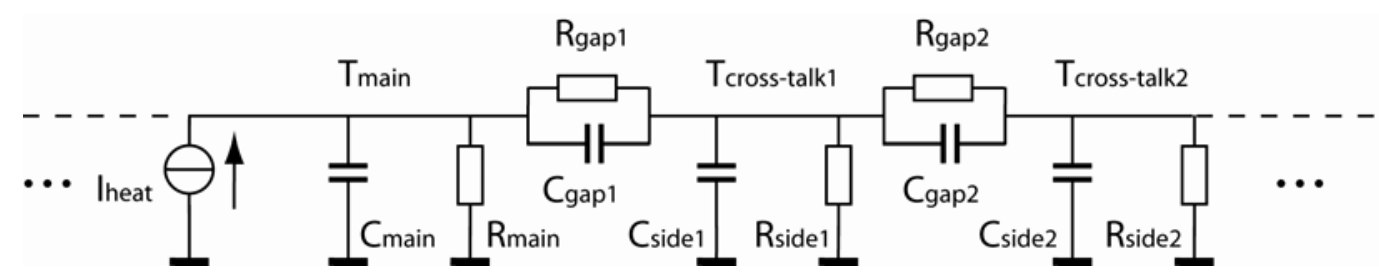

Figure 2 Lumped elements represent three TE elements in the electrical analogy.

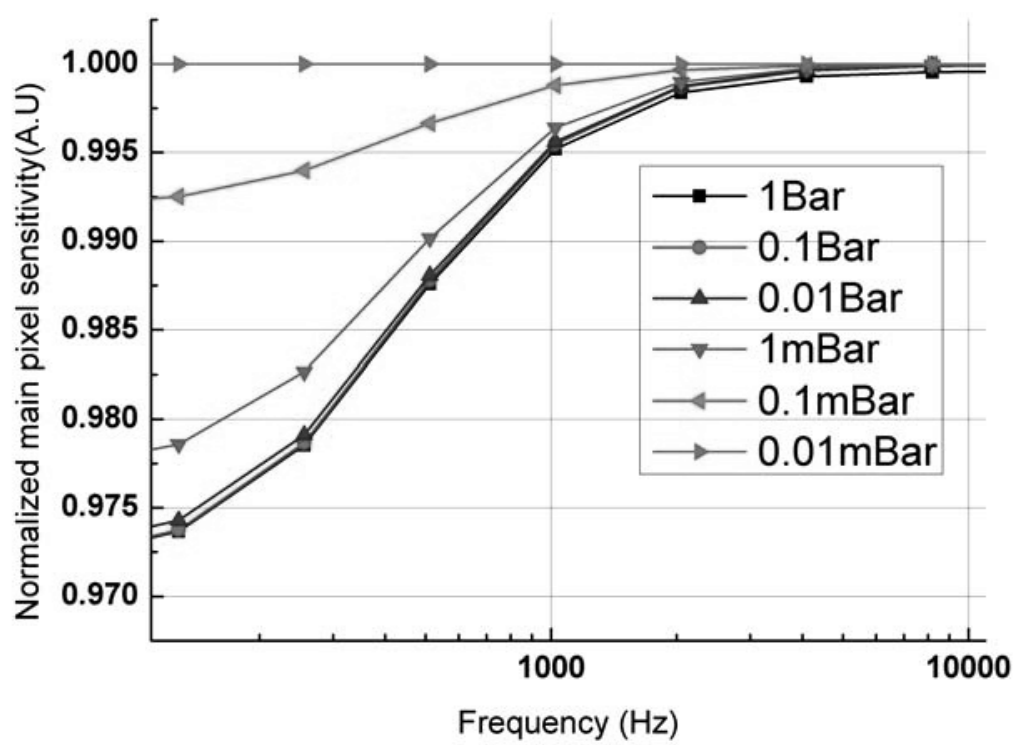

Figure 3 Thermal response of the main pixel as a function of packaging pressure (Normalized to $10^{-5}$ Bar pressure). 


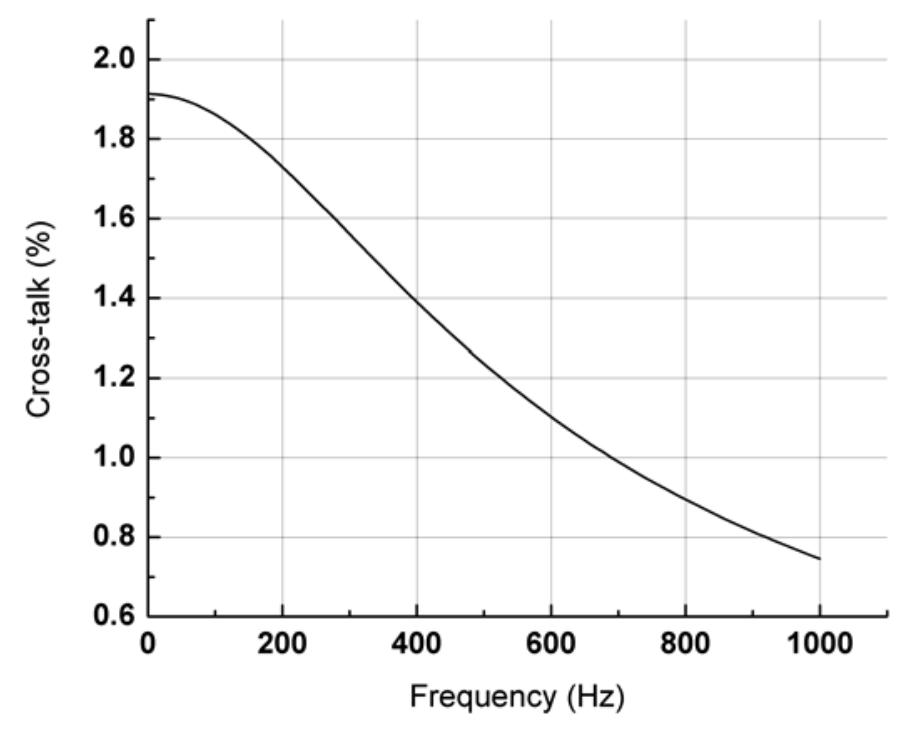

Figure 4 Thermal modulus plot of the cross-talk at $0.1 \mathrm{mBar}$ pressure.

\section{Experimental results}

For measuring the dynamic thermal response and thermal cross-talk, the laser spot is frequency modulated. The measurement setup includes a He-Ne laser, a optical chopper, a vacuum chamber, lockin amplifier SR830 for measuring thermal modulus plot and a digital oscilloscope for recording the thermal time constant to validate the $\mathrm{AC}$ response. The thermal modulus plot of three elements with the heated (illuminated) pixel in the middle under the vacuum pressure of $0.1 \mathrm{mBar}$ is shown in the Fig $5(\mathrm{a})$. The measured cross-talk measured is $1.5 \%$, which is in agreement with simulation. The cut-off frequency of the main pixel and side pixel in the measurement of atmosphere pressure is $240 \mathrm{~Hz}$ and $183 \mathrm{~Hz}$ respectively. This $-3 \mathrm{~dB}$ point has been validated with $\mathrm{RC}$ time measurement using the oscilloscope. The cut-off frequency of the side pixel is lower than the heated pixel since the side pixel is away from heat source and is subjected to more thermal capacitance. The ratio of cut-off frequency in experiment of heated to side element is 0.76 , which also is in agreement with the simulation result $(0.76)$.

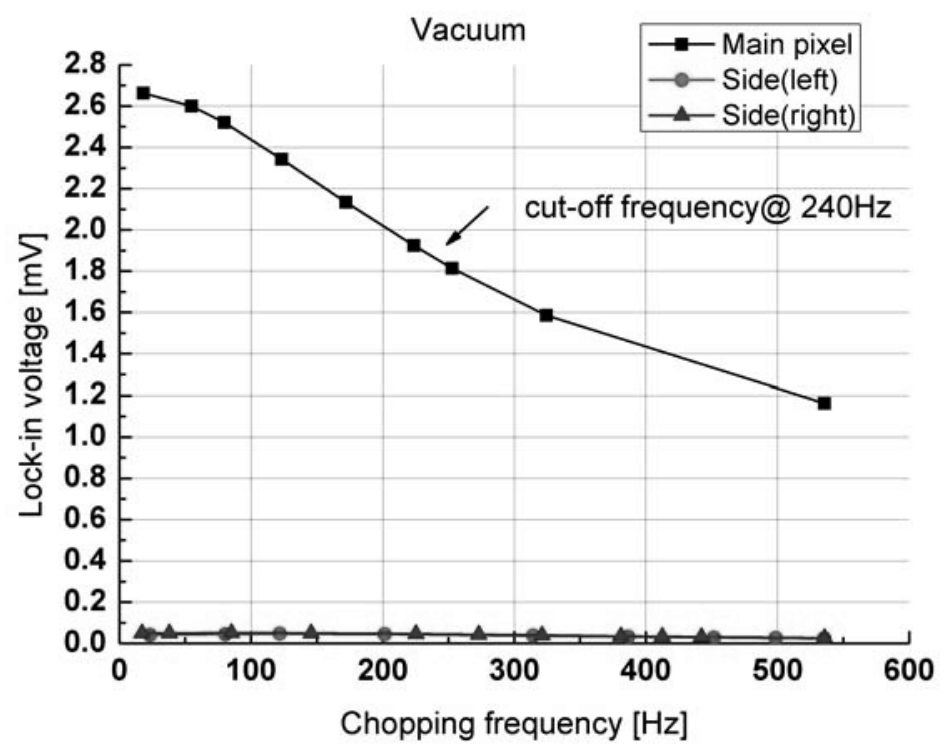

(a) 


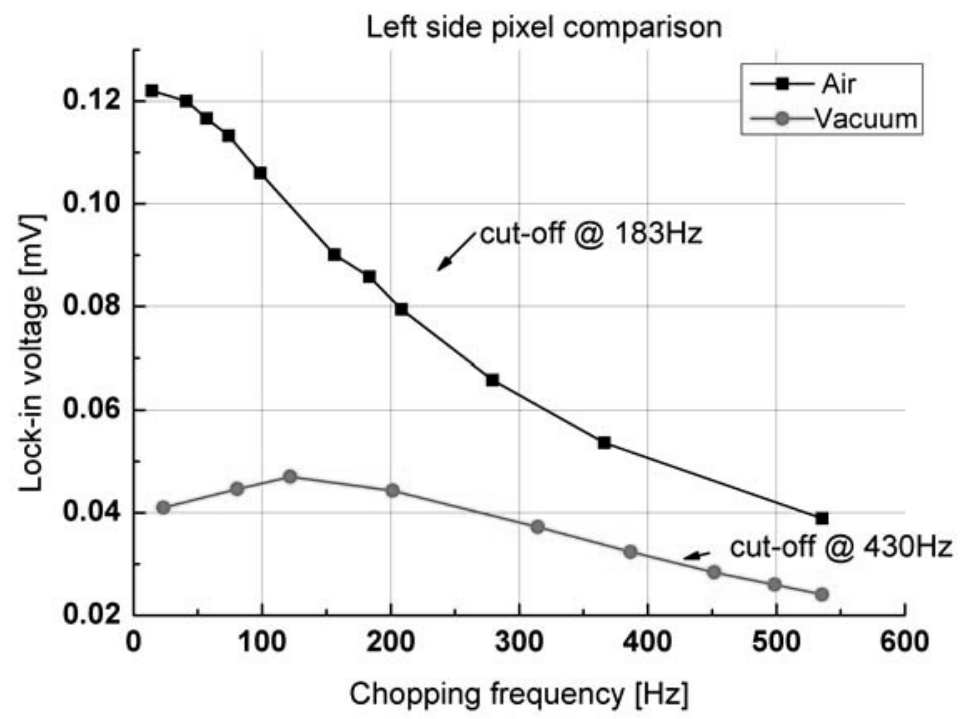

(b)

Figure 5 (a) Thermal modulus plot of three pixels under the $0.1 \mathrm{mBar}$ vacuum pressure. (b) Comparison of side pixel in normal and $0.1 \mathrm{mBar}$ pressure.

\section{Conclusions}

Cross-talk analysis and experimental results of a CMOS compatible thermopile array for use in a miniaturized infrared micro-spectrometer have been presented. The dynamic response of three neighboring pixels has been analyzed and is validated with the experimental results. It indicates either decreasing the package pressure or wider with gap between elements in order to further reduce the cross talk. A wider trench between elements helps to decrease cross-talk, but not efficiently in terms of optical resolving power. Moreover, the larger element pitch in the array would reduce the spectral resolution. A more effective solution is to operate the array at reduced air pressure, which also contributes to the sensitivity of each thermopile element.

\section{References}

[1] H. Wu, S. Grabarnik, A. Emadi, G. de Graaf and R.F. Wolffenbuttel, "A thermopile detector array with scaled TE elements for use in an integrated IR micro-spectrometer", J. Micromech. Microeng. 18 064017, 2008.

[2] Fluid Flow Databook, General Electric, May 1982, Section 410.2.

[3] F. Volklein, H. Baltes, "Optimization tool for the performance parameters of thermoelectric microsensors", Sens. Actuators A 36 65-71, 1993.

[4] C. Escriba, E. Campo, D. Esteve, J.Y. Fourniols, "Complete analytical modeling and analysis of micromachined thermoelectric uncooled IR sensors", Sens. Actuators A 120 (1) 267-276, 2005.

[5] D. Pitts and L. Sissom, Schaum's Outline of Theory and Problems of Heat Transfer. New York: McGraw-Hill, 1998. 